\title{
Formation of precise 2D Au particle arrays via thermally induced dewetting on pre-patterned substrates
}

\author{
Dong Wang ${ }^{*}$, Ran $\mathrm{Ji}^{2}$ and Peter Schaaf ${ }^{* 1}$
}

\section{Full Research Paper}

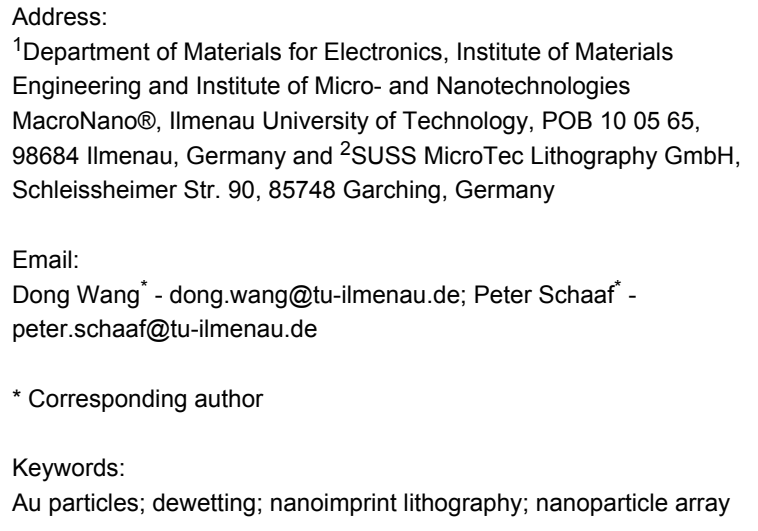

Beilstein J. Nanotechnol. 2011, 2, 318-326. doi:10.3762/bjnano.2.37

Received: 16 February 2011

Accepted: 03 June 2011

Published: 22 June 2011

Associate Editor: P. Leiderer

(C) 2011 Wang et al; licensee Beilstein-Institut. License and terms: see end of document.

\begin{abstract}
The fabrication of precise 2D Au nanoparticle arrays over a large area is presented. The technique was based on pre-patterning of the substrate before the deposition of a thin Au film, and the creation of periodic particle arrays by subsequent dewetting induced by annealing. Two types of pre-patterned substrates were used: The first comprised an array of pyramidal pits and the second an array of circular holes. For the dewetting of Au films on the pyramidal pit substrate, the structural curvature-driven diffusion cooperates with capillarity-driven diffusion, resulting in the formation of precise 2D particle arrays for films within a structure dependent thickness-window. For the dewetting of Au films on the circular hole substrate, the periodic discontinuities in the films, induced by the deposition, can limit the diffusion paths and lead to the formation of one particle per individual separated region (holes or mesas between holes), and thus, result in the evolution of precise $2 \mathrm{D}$ particle arrays. The influence of the pre-patterned structures and the film thickness is analyzed and discussed. For both types of pre-patterned substrate, the Au film thickness had to be adjusted in a certain thickness-window in order to achieve the precise 2D particle arrays.
\end{abstract}

\section{Introduction}

An increasing amount of scientific attention is being paid to the ordered arrangement of metallic nanoparticles, due to their wide range of potential applications in plasmonics [1,2], magnetic memories [3], DNA detection [4], and catalysis for nanowire and nanofiber growth [5,6]. Nanoparticle arrays are typically fabricated either by chemical processes based on self-assembly or by lithography based nanostructuring. The synthesis of 3D arrays of ligand stabilized Au nanoparticles using the selfassembly method has been reported [7], and electron beam lithography has also been used to define positioned nanoparticles at low throughput [8]. Another simple method for the formation of nanoparticle arrays is based on the dewetting process of 
thin metal films on an inert substrate. Dewetting of metallic films on a substrate is driven by the reduction of the surface energy of the thin film and of the interface energy between the film and substrate, and can be induced by thermal annealing [9-11], pulsed laser heating [12-20], ion irradiation [21-24], and electron irradiation [25]. Dewetting proceeds by surface diffusion even in the solid state well below the melting temperature of the film [9-11]. In addition, metals such as $\mathrm{Ni}, \mathrm{Ag}, \mathrm{Co}$, and $\mathrm{Au}$ have a weak interaction with $\mathrm{SiO}_{2}$ substrate, which results in low activation energy for metal atom migration [12].

Dewetting is a well known spontaneous physical phenomenon describing the rupture of a thin liquid film on a substrate and the formation of droplets. Dewetting dynamics of liquid polymer films have been studied [26-29] and three dewetting mechanisms are known: (1) Heterogeneous nucleation, which initiates from a defect located at the film surface or the film-substrate interface, (2) homogeneous nucleation, which occurs via a small thermal density fluctuation that acts as a nucleus for hole formation, and (3) spinodal dewetting, which occurs by the amplification of periodic film thickness fluctuations (i.e., capillary wave); such films induce self-correlated dewetting patterns [25].

Recently, dewetting of solid films has also been studied. Theoretically, for the defect-free and homogenous films, the surface energy driven mechanism starts at the film boundary, with edge agglomeration via capillary edge instability, and is then followed by particle formation via Rayleigh instability [30,31]. However, real films comprise defects and fluctuations in the film thickness. As well as by edge agglomeration [32,33], voids can nucleate due to periodic film thickness fluctuations (spinodal dewetting), or at defects, which is then followed by void growth and particle formation [31]. For polycrystalline metallic films, dewetting is also affected by the character of grain boundaries [33]. Altogether, dewetting of polycrystalline metallic films on a flat substrate usually leads to a broad distribution of particle size and spacing.

On the other hand, dewetting of metallic films on pre-patterned substrates can lead to the formation of ordered particle arrays. Formation of 2D ordered arrays of nanoparticles was observed on a thin metal film that had been patterned using focused ion beam (FIB) before the dewetting process [34]. However, the FIB patterning is a time-consuming process. Giermann and Thompson reported the formation of a 2D ordered Au nanoparticle array, with uniform size and aligned crystallographic orientation, on a substrate with an array of periodic pits, via solid-state dewetting induced by annealing at $850{ }^{\circ} \mathrm{C}$ [9]. Our previous work showed that a pre-patterned substrate with deep grid grooves can also lead to the formation of a $2 \mathrm{D}$ ordered $\mathrm{Au}$ nanoparticle array via dewetting induced by annealing at $900{ }^{\circ} \mathrm{C}$ [11]. Elsewhere, a 2D ordered Au nanoparticle array was formed on a nano-hole patterned substrate via electron-beaminduced dewetting of the Au thin film [25]. Ripple patterned $\mathrm{SiO}_{2}$ substrates [35] and stepped alumina substrates [36] also led to the formation of metallic nanoparticle arrays via thermal dewetting. The explanation for this is that the structural curvature and the corresponding chemical potential is modulated by the pre-patterned substrate structure, and thereby the ordered particle array is prone to evolve $[9,11,25,35,36]$. Here, thermal dewetting of the Au films. induced by annealing, has been studied, on both the flat substrate and two types of pre-patterned substrates (one with an array of pyramidal pits and one with an array of circular holes, made using nanoimprint lithography), and large areas of 2D ordered nanoparticle arrays were fabricated. Instead of the mechanism based on the modulation of chemical potential, as presented in the previous work, it is found here that the deposition-induced periodic discontinuities of the Au films, on the substrate with an array of circular holes, limited the diffusion path and resulted in the formation of $2 \mathrm{D}$ ordered nanoparticle arrays with well defined particle size and spacing more effectively.

\section{Results}

$\mathrm{Au}$ films were deposited on flat, and two types of pre-patterned, $\mathrm{SiO}_{2} / \mathrm{Si}$ substrates for the thermal dewetting induced by an annealing process at $900{ }^{\circ} \mathrm{C}$ in $\mathrm{N}_{2}$. Both types of pre-patterned substrates were fabricated using substrate conformal imprint lithography (SCIL) [37] and investigated by scanning electron microscopy (SEM): One with a square array of pyramidal pits (substrate type A), shown in Figure 1a, and another with an array of circular holes with square symmetry (substrate type B), shown in Figure 1b. The pits in substrate A have a spatial period of $513 \mathrm{~nm}$ and a depth of $150 \mathrm{~nm}$. The holes in the substrate B have the same spatial period of $513 \mathrm{~nm}$, a diameter of about $490 \mathrm{~nm}$, and a depth of $120 \mathrm{~nm}$.

Figure 2 shows the SEM images of the Au particles formed from the $5 \mathrm{~nm}$ and $60 \mathrm{~nm}$ thick $\mathrm{Au}$ films on a flat $\mathrm{SiO}_{2} / \mathrm{Si}$ substrate. Usually, flat substrates lead to a broad distribution of particle size and spacing of the dewetted particles. Figure $2 \mathrm{c}$ shows the particle size distributions produced by dewetting of $\mathrm{Au}$ films with thicknesses from $5 \mathrm{~nm}$ to $60 \mathrm{~nm}$ on the flat substrates. Both, mean particle size $<d_{\mathrm{p}}>$ and the width of particle size distribution $\sigma_{\mathrm{p}}$ increase with increasing film thickness.

Figure 3 shows the SEM images of the Au particles produced from the $10 \mathrm{~nm}, 20 \mathrm{~nm}, 40 \mathrm{~nm}$, and $60 \mathrm{~nm}$ thick Au films on the substrate A (pyramidal pits). For the $10 \mathrm{~nm}$ thick film, several particles could be observed in any one pit. Furthermore, there were relatively more larger size particles located in the pits than 

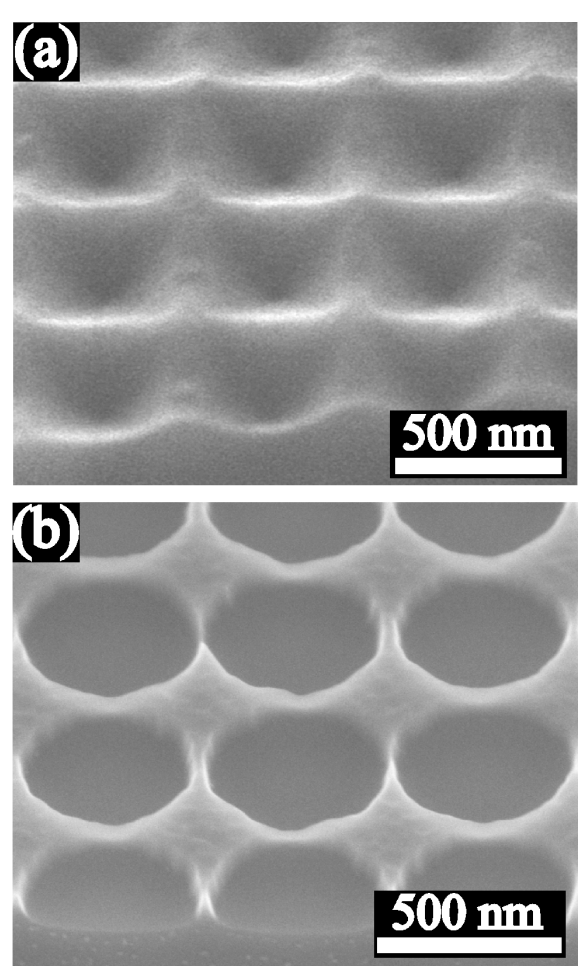

Figure 1: SEM images at $45^{\circ}$ tilt of (a) a square array of pyramidal pits (substrate $A$ ) and (b) an array of circular holes with square symmetry (substrate B) on the ridges between pits, as seen in Figure $3 \mathrm{a}$. For the $20 \mathrm{~nm}$ thick film, all particles were located in the pits and in every pit there was only one particle. Thus a periodic array of particles evolved as seen in Figure 3b. For the $40 \mathrm{~nm}$ thick film, some pits are empty, while the size of some particles is clearly larger than the spatial period of the pyramidal pits and sometimes even two pits were occupied by one large particle, as seen in Figure 3c. For the $60 \mathrm{~nm}$ thick film, many more pits are empty and more large individual particles occupying two or more pits are formed, as seen in Figure 3d.

The influence of substrate B (circular holes) on the dewetting was somewhat different. Figure 4 shows the SEM images of the dewetted $\mathrm{Au}$ particles on the substrate $\mathrm{B}$, which were formed from the $10 \mathrm{~nm}, 20 \mathrm{~nm}, 40 \mathrm{~nm}$, and $60 \mathrm{~nm}$ thick Au films. For the $10 \mathrm{~nm}$ thick film, several particles were often observed in one individual hole, and for the most part, only one particle with similar size was located on each of the mesas between holes (Figure 4a). Further SEM investigation showed that the $10 \mathrm{~nm}$ thick as-deposited film contained a number of substrateexposing grooves. These grooves play an important role for the dewetting process. Au films retract from the edge of the grooves and the grooves expand, such that multiple particles are formed per pit (Figure 3a) or per hole (Figure 4a). For the $20 \mathrm{~nm}$ thick Au film, there was only one particle formed in every hole and one on every mesa (Figure $4 b$ ). The particles in
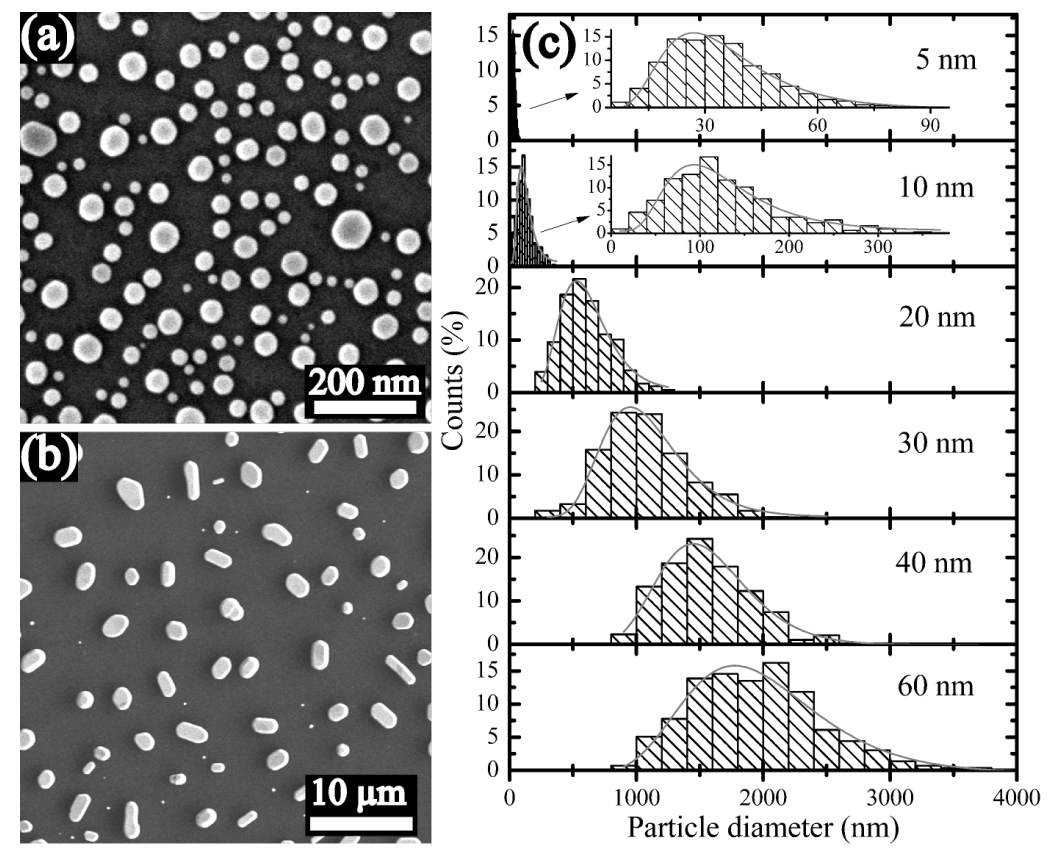

Figure 2: SEM images of induced particles on the flat $\mathrm{SiO}_{2} / \mathrm{Si}$ substrate after dewetting of the $5 \mathrm{~nm}$ (a) and $60 \mathrm{~nm}$ (b) thick Au films. (c) Histograms of particle size distributions produced by the dewetting of the $5 \mathrm{~nm}, 10 \mathrm{~nm}, 20 \mathrm{~nm}, 30 \mathrm{~nm}, 40 \mathrm{~nm}$, and $60 \mathrm{~nm}$ thick Au films on the flat SiO $2 / \mathrm{Si}$ substrates. Inset plots in (c) are magnified for clarity. Fitting curves (log-normal function) are superimposed on the histograms. 

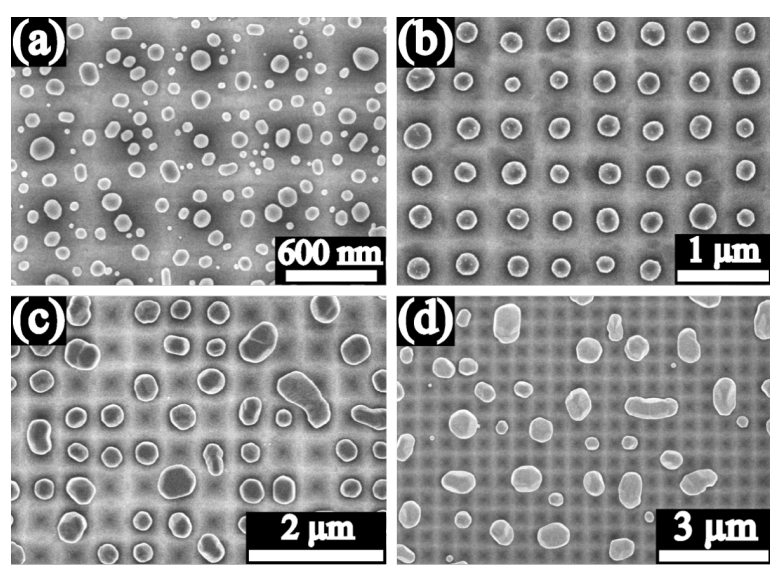

Figure 3: SEM images of Au particles produced on the $10 \mathrm{~nm}$ (a) $20 \mathrm{~nm}$ (b), $40 \mathrm{~nm}$ (c), and $60 \mathrm{~nm}$ (d) thick Au films on substrate $A$ (pyramidal pits).

the holes are clearly larger than the particles on the mesas. In addition, particle chains consisting of much smaller particles were formed on the circular interior walls of the holes for the cases of the $10 \mathrm{~nm}$ and $20 \mathrm{~nm}$ thick Au films (Figure 4a and b). For the $40 \mathrm{~nm}$ thick Au film, similarly, only one particle was formed in every hole and on every mesa (Figure 4c), but particle chains around the interior walls of the holes were not observed. For the case of the $60 \mathrm{~nm}$ thick Au film, most individual holes were filled with one particle, but on most mesas there was no particle located (Figure 4d).
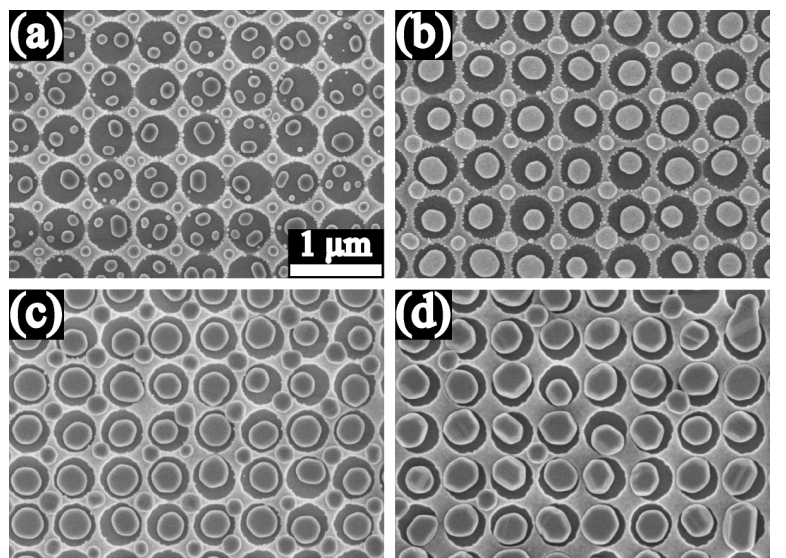

Figure 4: SEM images of Au particles produced from the $10 \mathrm{~nm}(\mathrm{a})$, $20 \mathrm{~nm}$ (b), $40 \mathrm{~nm}$ (c), and $60 \mathrm{~nm}$ (d) thick Au films on substrate B (circular holes). The scale bar $1 \mu \mathrm{m}$ is valid for all 4 images.

Figure 5 shows the magnified SEM images at $30^{\circ}$ tilt of the $20 \mathrm{~nm}$ and $40 \mathrm{~nm}$ thick as-deposited Au films, and the corresponding dewetted particles on the substrate $\mathrm{B}$. On the interior walls of the holes, discontinuity can be observed in both the
$20 \mathrm{~nm}$ and $40 \mathrm{~nm}$ thick as-deposited Au films (Figure 5a and c). The discontinuity morphology on the interior walls also changes with film thickness. When the film is initially very thin, the discontinuity region consists of isolated islands, for example in the $10 \mathrm{~nm}$ and $20 \mathrm{~nm}$ thick as-deposited films (Figure 5a). During dewetting, the isolated islands retract into small particles and thereby the chains of small particles can evolve (Figure 5b). As the thickness increases, the discontinuity region changes in form and consists of substrate-exposing grooves (Figure $5 \mathrm{c}$ ). The films retract from the groove edges, such that no small particles remain on the interior walls after dewetting (Figure 5d). The mean width of grooves decreases with increasing film thickness. Finally, if the thickness was increased further, the discontinuity on the interior walls should disappear.
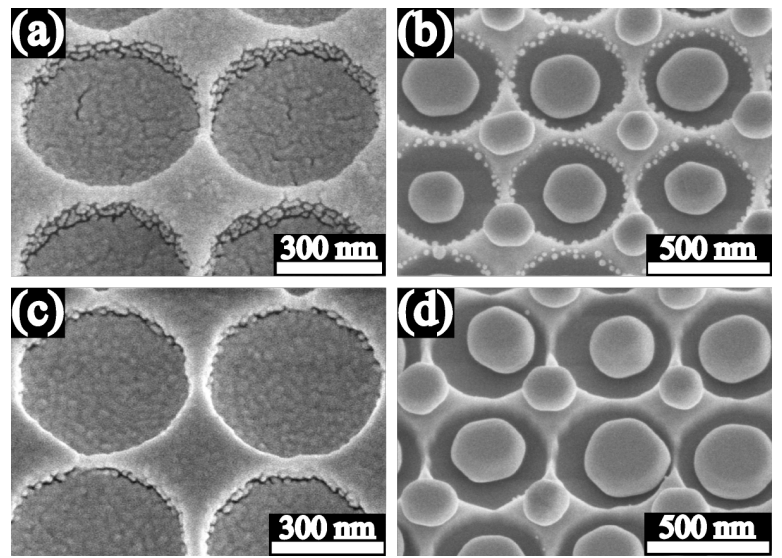

Figure 5: SEM images at $30^{\circ}$ tilt of the $20 \mathrm{~nm}$ (a) and $40 \mathrm{~nm}$ (c) thick as-deposited Au films, and the corresponding particles (b) and (d) after dewetting on substrate B (circular holes).

Figure 6 shows the particle size distributions produced by dewetting of the $20 \mathrm{~nm}$ thick Au films on the different substrates. Comparing to the flat substrate (Figure 6e), both prepatterned substrates A and B (Figure 6c and d) led to a clear reduction of absolute particle size and the width of the particle size distribution, i.e., the particle size is became smaller and more uniform. For a given film thickness, the particle size for the evolved particle arrays on both substrate A and substrate B is related to the individual structure dimension. Substrate A results in particle size distributions with clearly sharper peaks than substrate $B$, on which dewetted particles locate in two different regions: In the holes and on the mesas. Particle size distributions for particles in the holes (Figure 6b) and on the mesas (Figure 6a) of the substrate B are also plotted for comparison, and it is clear that the particles formed in holes generally are larger. 


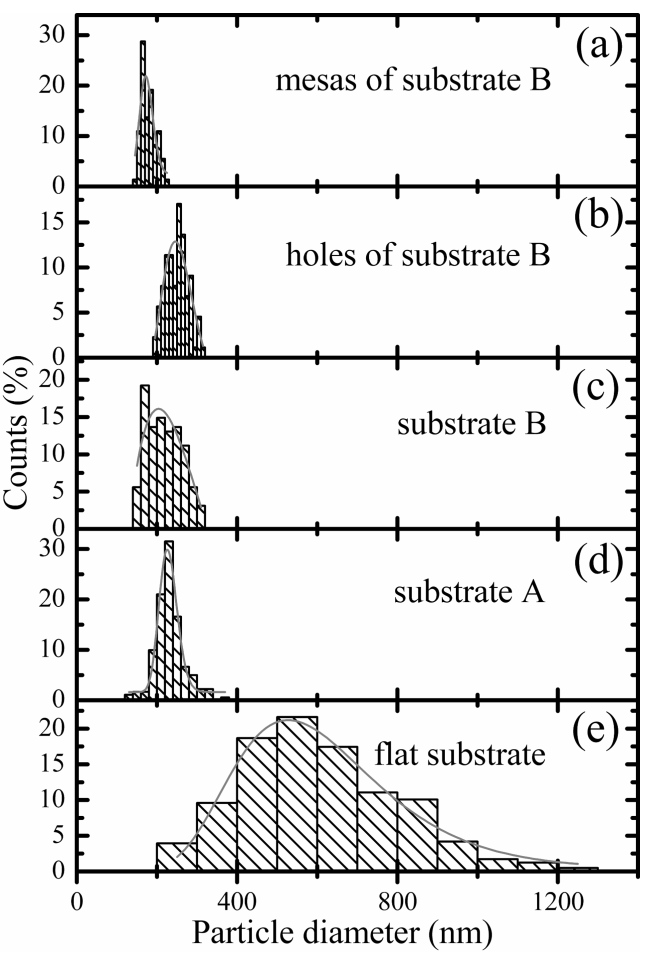

Figure 6: Histograms of particle size distributions, produced by dewetting of the $20 \mathrm{~nm}$ thick Au films, on the mesas of the substrate $B(a)$, in the holes of the substrate $B(b)$, on the substrate $B(c)$, on the substrate $A(d)$, and on the flat substrate (e). Fitting curves (fitted with lognormal function) are superimposed on the histograms.

Figure 7 displays the radially averaged autocorrelation function of Au particles dewetted from the $20 \mathrm{~nm}$ thick Au films on the different substrates. The radially averaged autocorrelation is

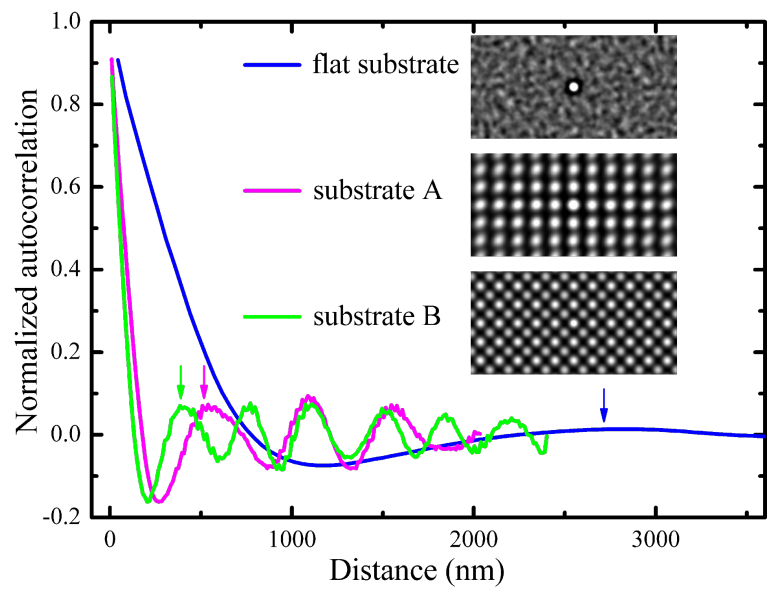

Figure 7: Plot of radially averaged autocorrelation of the induced particles of the $20 \mathrm{~nm}$ thick Au films on the flat substrate and on substrates $A$ and $B$. Arrows indicate the corresponding characteristic particle spacing $s$ (at maximum). Insets show the corresponding autocorrelation images. calculated from the autocorrelation (also known as pair correlation) of pixels of a binary image as a function of their radial distance. The first minimum of this function gives the information on particle size, and the subsequent first maximum indicates the characteristic length (particle spacing). Comparing the plot of particles on the flat substrate, the plots of particles on both prepatterned substrate A and B show a more periodic shape, indicating the high regularity of the nanoparticle arrays, which is also well confirmed by the autocorrelation images (insets in Figure 7). Characteristic particle spacing was determined based on the plan view SEM images and plotted as a function of the film thickness in Figure 8. Figure 8a shows the plot of mean particle size as a function of the film thickness. A similar trend is observed for the particles produced either on the flat substrate, or on the pre-patterned substrates, i.e., both mean particle size and characteristic particle spacing increase with increasing film thickness. In addition, both pre-patterned substrates A and B result in a clear reduction of the mean particle size and characteristic particle spacing as compared to the flat substrate. For film thickness below $20 \mathrm{~nm}$, both mean particle size and characteristic particle spacing for the particles on substrate A are smaller than those for particles on substrate B.

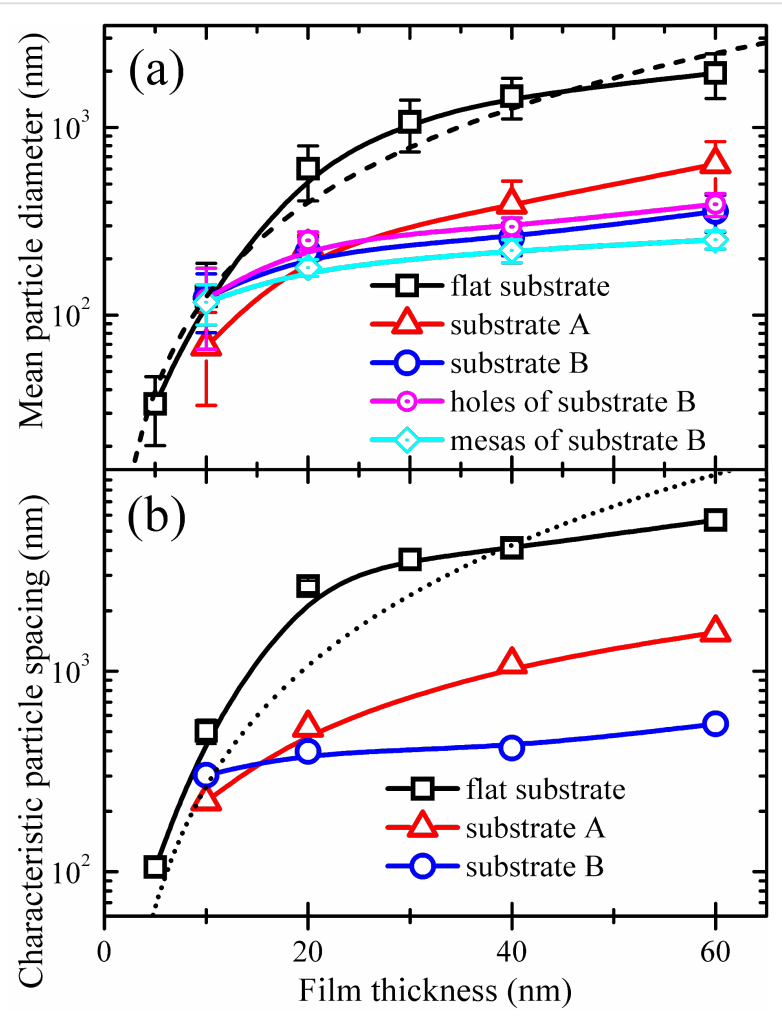

Figure 8: Plots of (a) the mean particle diameter and (b) the characteristic particle spacing as a function of the film thickness. The dashed line in (a) and the dotted line in (b) are the best fits for the flat substrate based on the variation of the mean particle size $\left\langle d_{p}\right\rangle$ and characteristic particle spacing $s$ with the film thickness $t$ as $\left\langle d_{p}\right\rangle \propto t^{5 / 3}$ and $s \propto t^{2}[14,18,38]$ 
However, from $20 \mathrm{~nm}$, on the contrary, both mean particle size and characteristic particle spacing for the particles on substrate A are larger than those for the particles on substrate B. It is also interesting to note that the characteristic particle spacing for the particles on substrate B first increases from $10 \mathrm{~nm}$ to $20 \mathrm{~nm}$, then almost stays constant between $20 \mathrm{~nm}$ and $40 \mathrm{~nm}$, and finally, increases from $40 \mathrm{~nm}$ to $60 \mathrm{~nm}$. The characteristic particle spacing for the dewetted particles of the $20 \mathrm{~nm}$ and $40 \mathrm{~nm}$ thick Au films on substrate B (397 nm and $414 \mathrm{~nm}$ ) should approximately equal the projected distance between the center of a hole and the center of the next mesa $(363 \mathrm{~nm})$, because there is typically only one particle in each hole and on each mesa. The characteristic particle spacing for the dewetted particles of the $20 \mathrm{~nm}$ thick Au film on substrate A and the $60 \mathrm{~nm}$ thick Au film on substrate B (522 nm and $546 \mathrm{~nm}$ ) should equal the spatial period of the substrate structures $(513 \mathrm{~nm})$. However, there are deviations of these characteristic particle spacings within $10-40 \mathrm{~nm}$, and this is probably due to the uncertainty of the radially averaged autocorrelation.

\section{Discussion}

The presented results show that the characteristics of the dewetted particles (particle size and spacing) depend on the film thickness, and that the pre-patterned substrates lead to a reduction of particle size and spacing, and result in the formation of precise $2 \mathrm{D}$ particle arrays via dewetting. In addition, the substrate conformal imprint lithography (SCIL) technique enables the production of large areas of pre-patterned substrates with high uniformity and the corresponding fabrication of large areas of ordered 2D particle arrays. However, the respective influence of two substrate structures on the formation of particle arrays is different.

\section{Dewetting of the Au films on the flat substrate}

According to the linear hydrodynamic spinodal dewetting theory for liquid films, the characteristic particle spacing $s$ and the mean particle size $<d_{\mathrm{p}}>$ vary with the film thickness $t$ as $s$ $\propto t^{2}$ and $<d_{\mathrm{p}}>\propto t^{5 / 3}[14,18,38]$. Based on these variations, the best fit for the particle spacing $s$ (dotted line in Figure $8 \mathrm{~b}$ ) doesn't conform well to the experimental data, indicating that the solid-state dewetting of the polycrystalline films is different from the mentioned theory and may be more complicated, despite the good agreement between the best fit (dashed line in Figure 8a) and experimental data for the particle diameter $<d_{\mathrm{p}}>$ on the flat substrate. Usually, the dewetting process of the solid films starts with void nucleation, and then proceeds with void growth and particle formation. For polycrystalline films on the flat substrates, void nucleation is generally thought to occur due to grain boundary grooving, via surface diffusion at the grain boundaries, and grain boundary triple junctions which intersect the substrate surface $[39,40]$. Recently, Mueller and Spolenak have reported that holes (large substrate-exposing voids) were found to protrude into the film predominantly at high angle grain boundaries during dewetting [41]. During annealing, grain boundary grooving and grain growth are competing kinetic processes. Grain growth is driven by the reduction of total grain boundary energy. The total grain boundary energy decreases with decreasing total grain boundary area. Microscopically, the reduction of the grain boundary area is accomplished by the movement of the individual boundaries and reducing the boundary curvature. To a first approximation, effective grooving will not occur during the rapid boundary moving [42], and even at high temperature, which facilitates surface diffusion, the effective grooving should be also retarded due to the upsetting of the rapid boundary movement. However, it has been shown that the velocity of boundary movement will decrease when the grain size reaches about three times the film thickness [43]. Subsequently, grooving occurs and in turn the formed grooves suppress the further boundary motion [40]. The mean diameter of the grown grains and the characteristic spacing of grain boundary triple junctions, which scale with the particle size and spacing for the dewetted particles in an approximate manner, increase with the film thickness. So it is expected that the nucleated void density decreases with increasing film thickness. In addition, an experimental investigation from Jiran and Thompson has shown that the void growth rate $R_{\mathrm{V}}$ decreases with increasing film thickness $t$ dramatically $\left(R_{\mathrm{v}} \propto t^{-3}\right)$ [32]. Therefore, it is reasonable that the particle size and particle spacing increases with film thickness (Figure 8).

\section{Dewetting of the Au films on the substrate A (pyramidal pits)}

Dewetting of the solid films is driven by the reduction of the surface energy (capillarity driven dewetting mechanism) and results in the formation of particles via surface diffusion. Surface curvatures of periodic substrate structures are associated with the chemical potential, introducing an additional driving force for the diffusion from the position with positive local curvature (peaks or ridges) to the position with negative local curvature (valleys), and an additional barrier for the diffusion crossing the position with positive local curvature (peaks or ridges) $[9,11,25,35,36]$. This is schematically shown in Figure 9. The occurrence of film thinning and subsequent film rupture or void nucleation at the peaks or ridges is expected due to the curvature driving diffusion, and the kinetics of this process depend on the film thickness for the structures with a fixed curvature. The formation of the precise particle array with one particle per pit on the substrate A (Figure 3b) confirms the influence of the periodic structure with curvature on the dewetting process. But the curvature driving diffusion is not the only process operating, and this process combined with the capil- 


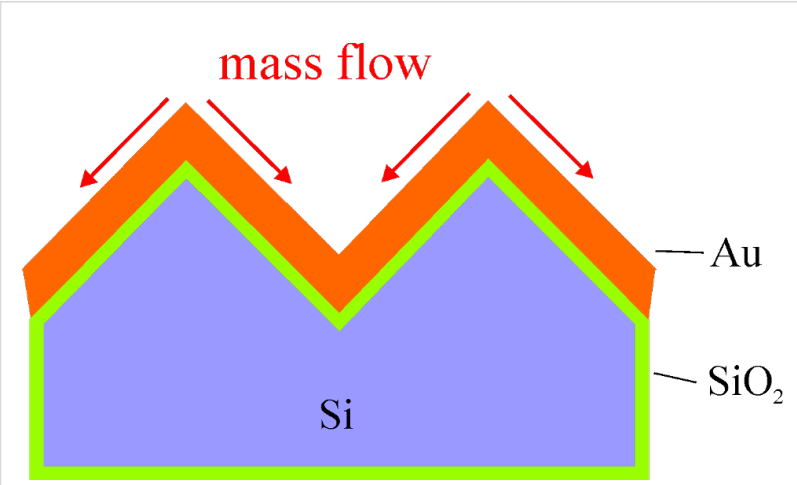

Figure 9: Schematic illustration of the mass flow induced by the local curvature driving diffusion on the substrate $A$.

larity driven process and the grain growth leads to the formation of the precise particle array with one particle per pit only in the $20 \mathrm{~nm}$ thick Au film. However, the particle size and spacing are reduced clearly due to the modulation of periodic structure with curvature.

\section{Dewetting of the Au films on the substrate B (circular holes)}

Comparing to the substrate $\mathrm{A}$, the substrate $\mathrm{B}$ has some different influences on the dewetting, resulting in the formation of the precise particle arrays with one particle per hole and/or per mesa in the $20 \mathrm{~nm}, 40 \mathrm{~nm}$, and even $60 \mathrm{~nm}$ thick Au films. The interior sidewalls of the holes of the substrate B are perpendicular to the substrate surface, and the consequence is the evolution of the film discontinuity at the perpendicular interior sidewalls by the film deposition (Figure $5 \mathrm{a}$ and c). The film discontinuity is substrate-exposing, separates the film and limits the diffusion pathway within the individual regions (holes and mesas). The Au retracts from the discontinuity regions and one particle is formed in every individual region after the annealing, so that the precise particle arrays are evolved (Figure $4 \mathrm{~b}-\mathrm{d}$ ). Figure $4 \mathrm{~b}$ and $\mathrm{c}$ even shows the possibility to produce the precise $3 \mathrm{D}$ particle arrays by controlling the depth and areas of the holes and the area of the mesas.

Assuming that the particles are hemispherical, that the as-deposited films have a similar density to their bulk counterparts and that there is no material loss during annealing, the size of particle on the mesas can be calculated for the given as-deposited film thickness. Due to the structural constraint, the maximal size of the particles on the mesas is limited by the mesa dimension; otherwise the particles would be not stable on the mesas. Therefore, there is a corresponding maximal as-deposited film thickness $\left(t_{\max }\right)$ for the stable particles on the mesas, as schematically presented in Figure 10. Consequently, the $t_{\max }$ for the mesas on the substrate $\mathrm{B}$ is calculated as about

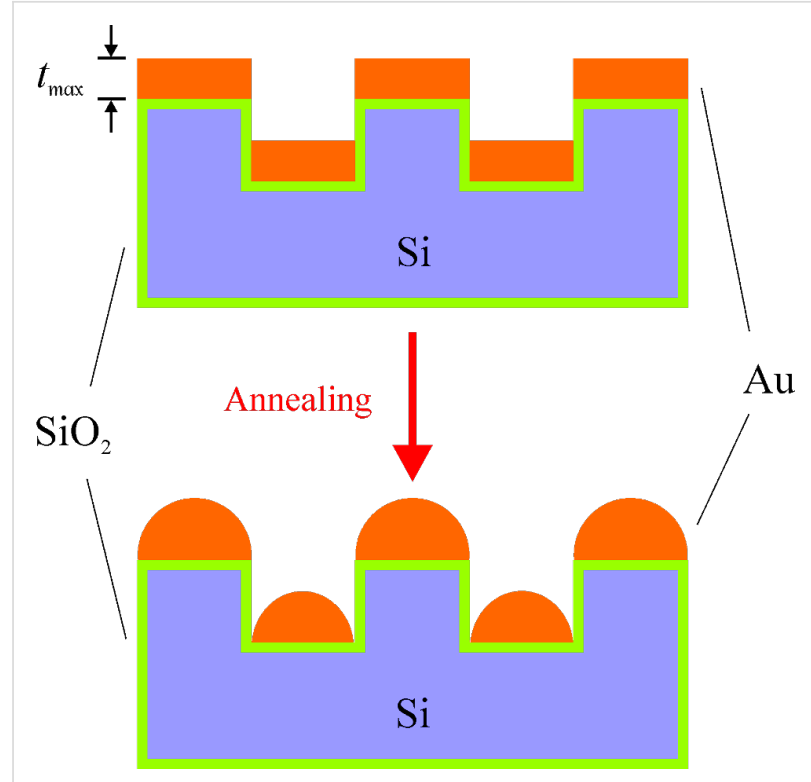

Figure 10: Schematic drawing of the particle formation on the substrate $B$ via annealing induced dewetting.

$45 \mathrm{~nm}$. Altogether, for the $60 \mathrm{~nm}$ thick film on the substrate B, particles are prone to be formed in the holes which have a much larger area.

\section{Conclusion}

We have studied the dewetting of Au films on the flat and prepatterned substrates and discussed the influence of the substrate structures on the formation and characteristics of the dewetted particles. The pre-patterned substrates result in a clear reduction in particle size and particle spacing. Substrate A and substrate B have different influences on the dewetting, and both can lead to the formation of the precise 2D Au nanoparticle arrays. For that, the film thickness has to be adjusted to a structure-dependent value or thickness-window (around $20 \mathrm{~nm}$ for the substrate A, and ideally $20-40 \mathrm{~nm}$ for the substrate B). In addition, the possibility to fabricate precise $3 \mathrm{D}$ particle arrays is as well indicated by controlling the structure parameters of the substrate type B. The remarkable optical and plasmonic properties of the noble metallic particles indicate the potential applicability of this method in fabricating large areas of particle arrays for the plasmonic devices or in improving the efficiency of the photovoltaic devices and light-emitting diodes (LED) by modification of the surface optical properties.

\section{Experimental}

The surface of (100) Si wafers was pre-patterned into a square array of pyramidal pits (substrate type A), shown in Figure 1a, and an array of circular holes with square symmetry (substrate type B), shown in Figure 1b, by employing the substrate conformal imprint lithography (SCIL) and reactive ion etching 
(RIE, Oxford Plasmalab 100 and STS 320 PC). The SCIL technique, which was developed by Philips Research and SUSS MicroTec, combines the advantages of both UV nanoimprint lithography techniques with rigid stamp for the best resolution and with soft stamp for the large-area (6 inch area) patterning. Thermal oxide several nanometers thick was grown on the prepatterned Si to prevent reactions between the substrates and the subsequently deposited Au films. Additionally, $20 \mathrm{~nm}$ thick thermal oxides were also grown on a flat $\mathrm{Si}$ wafer for comparison. Au films with thicknesses $t$ of $5 \mathrm{~nm}, 10 \mathrm{~nm}, 20 \mathrm{~nm}, 30 \mathrm{~nm}$, $40 \mathrm{~nm}$ and $60 \mathrm{~nm}$ were deposited on the flat substrates and with thicknesses of $10 \mathrm{~nm}, 20 \mathrm{~nm}, 40 \mathrm{~nm}$ and $60 \mathrm{~nm}$ on the prepatterned substrates using electron beam evaporation at a base pressure of $2 \times 10^{-7} \mathrm{mbar}$. Film thicknesses were determined by the quartz crystal monitor and then verified by profilometer measurements (Dektak 150 - Veeco). After deposition, the films were annealed in pure $\mathrm{N}_{2}$ at $900{ }^{\circ} \mathrm{C}$ for $15 \mathrm{~min}$ in order to induce the dewetting. After a rapid initial heating from room temperature to $200^{\circ} \mathrm{C}$, it took $5 \mathrm{~min}$ for the further heating from $200{ }^{\circ} \mathrm{C}$ to $900{ }^{\circ} \mathrm{C}$. Dewetted particles were investigated using ultra-high resolution scanning electron microscopy (SEM, Hitachi S-4800). Particle sizes were calculated as circular diameters and determined using a threshold image contrast in the SEM images and performing a pixel count.

\section{Acknowledgements}

The authors are grateful to Manuela Breiter and Joachim Döll from Ilmenau University of Technology for performing reactive ion etching and deposition of the Au films.

\section{References}

1. Maier, S. A.; Brongersma, M. L.; Kik, P. G.; Meltzer, S.; Requicha, A. A. G.; Atwater, H. A. Adv. Mater. 2001, 13, 1501-1505. doi:10.1002/1521-4095(200110)13:19<1501::AID-ADMA1501>3.0.CO; $2-Z$

2. Fan, J. A.; Wu, C.; Bao, K.; Bao, J.; Bardhan, R.; Halas, N. J.; Manoharan, V. N.; Nordlander, P.; Shvets, G.; Capasso, F. Science 2010, 328, 1135-1138. doi:10.1126/science.1187949

3. Cheng, J. Y.; Ross, C. A.; Chan, V. Z.-H.; Thomas, E. L.; Lammertink, R. G. H.; Vancso, G. J. Adv. Mater. 2001, 13, 1174-1178. doi:10.1002/1521-4095(200108)13:15<1174::AID-ADMA1174>3.0.CO; 2-Q

4. Fritzsche, W.; Taton, T. A. Nanotechnology 2003, 14, R63-R73. doi:10.1088/0957-4484/14/12/R01

5. Kodambaka, S.; Tersoff, J.; Reuter, M. C.; Ross, F. M. Science 2007, 316, 729-732. doi:10.1126/science.1139105

6. Guan, Y. F.; Pearce, R. C.; Melechko, A. V.; Hensley, D. K.; Simpson, M. L.; Rack, P. D. Nanotechnology 2008, 19, 235604. doi:10.1088/0957-4484/19/23/235604

7. Schmid, G.; Simon, U. Chem. Commun. 2005, 697-710. doi:10.1039/b411696h

8. Hicks, E. M.; Zou, S.; Schatz, G. C.; Spears, K. G.; Van Duyne, R. P.; Gunnarsson, L.; Rindzevicius, T.; Kasemo, B.; Käll, M. Nano Lett. 2005, 5, 1065-1070. doi:10.1021/nl0505492
9. Giermann, A. L.; Thompson, C. V. Appl. Phys. Lett. 2005, 86, 121903. doi:10.1063/1.1885180

10. Kim, D.; Giermann, A. L.; Thompson, C. V. Appl. Phys. Lett. 2009, 95, 251903. doi:10.1063/1.3268477

11. Wang, D.; Schaaf, P. J. Mater. Sci.: Mater. Electron. 2010. doi:10.1007/s10854-010-0260-2

12. Henley, S. J.; Carey, J. D.; Silva, S. R. P. Phys. Rev. B 2005, 72, 195408. doi:10.1103/PhysRevB.72.195408

13. Favazza, C.; Trice, J.; Krishna, H.; Kalyanaraman, R.; Sureshkumar, R. Appl. Phys. Lett. 2006, 88, 153118. doi:10.1063/1.2195113

14. Trice, J.; Thomas, D.; Favazza, C.; Sureshkumar, R.; Kalyanaraman, R. Phys. Rev. B 2007, 75. doi:10.1103/PhysRevB.75.235439

15. Favazza, C.; Trice, J.; Kalyanaraman, R.; Sureshkumar, R. Appl. Phys. Lett. 2007, 91, 043105. doi:10.1063/1.2762294

16. Favazza, C.; Kalyanaraman, R.; Sureshkumar, R. J. Appl. Phys. 2007, 102, 104308. doi:10.1063/1.2812560

17. Krishna, H.; Miller, C.; Longstreth-Spoor, L.; Nussinov, Z.; Gangopadhyay, A. K.; Kalyanaraman, R. J. Appl. Phys. 2008, 103, 073902. doi:10.1063/1.2901198

18. Krishna, H.; Favazza, C.; Gangopadhyay, A. K.; Kalyanaraman, R. JOM 2008, 60, 37-42. doi:10.1007/s11837-008-0115-y

19. Kondic, L.; Diez, J. A.; Rack, P. D.; Guan, Y.; Fowlkes, J. D. Phys. Rev. E 2009, 79, 026302. doi:10.1103/PhysRevE.79.026302

20. Krishna, H.; Sachan, R.; Strader, J.; Favazza, C.; Khenner, M.; Kalyanaraman, R. Nanotechnology 2010, 21, 155601. doi:10.1088/0957-4484/21/15/155601

21. Hu, X.; Cahill, D. G.; Averback, R. S. Appl. Phys. Lett. 2000, 76, 3215-3217. doi:10.1063/1.126633

22. Hu, X.; Cahill, D. G.; Averback, R. S. J. Appl. Phys. 2001, 89, 7777-7783. doi:10.1063/1.1372623

23. Hu, X.; Cahill, D. G.; Averback, R. S. J. Appl. Phys. 2002, 92, 3995-4000. doi:10.1063/1.1503387

24. Hu, X.; Cahill, D. G.; Averback, R. S.; Birtcher, R. C. J. Appl. Phys. 2003, 93, 165-169. doi:10.1063/1.1527712

25. Kojima, Y.; Kato, T. Nanotechnology 2008, 19, 255605. doi:10.1088/0957-4484/19/25/255605

26. Seemann, R.; Herminghaus, S.; Jacobs, K. Phys. Rev. Lett. 2001, 86, 5534-5537. doi:10.1103/PhysRevLett.86.5534

27. Seemann, R.; Herminghaus, S.; Jacobs, K. Phys. Rev. Lett. 2001, 87, 196101. doi:10.1103/PhysRevLett.87.196101

28. Herminghaus, S.; Seemann, R.; Landfester, K. Phys. Rev. Lett. 2004, 93, 017801. doi:10.1103/PhysRevLett.93.017801

29. Sharma, A.; Verma, R. Langmuir 2004, 20, 10337-10345. doi:10.1021/la048669x

30. Kan, W.; Wong, H. J. Appl. Phys. 2005, 97, 043515. doi:10.1063/1.1845579

31. Danielson, D. T.; Sparacin, D. K.; Michel, J.; Kimerling, L. C. J. Appl. Phys. 2006, 100, 083507. doi:10.1063/1.2357345

32. Jiran, E.; Thompson, C. V. J. Electron. Mater. 1990, 19, 1153-1160. doi:10.1007/BF02673327

33. Jiran, E.; Thompson, C. V. Thin Solid Films 1992, 208, 23-28. doi:10.1016/0040-6090(92)90941-4

34. Lin, C. H.; Jiang, L.; Chai, Y. H.; Xiao, H.; Chen, S. J.; Tsai, H. L. Appl. Phys. A: Mater. Sci. Process. 2010, 98, 855-860. doi:10.1007/s00339-010-5552-0

35. Petersen, J.; Mayr, S. G. J. Appl. Phys. 2008, 103, 023520. doi:10.1063/1.2832758

36. Basu, J.; Carter, C. B.; Divakar, R.; Mukherjee, B.; Ravishankar, N. Appl. Phys. Lett. 2009, 94, 171114. doi:10.1063/1.3127442 
37. Ji, R.; Hornung, M.; Verschuuren, M. A.; van de Laar, R.; van Eekelen, J.; Plachetka, U.; Moeller, M.; Moormann, C. Microelectron. Eng. 2010, 87, 963-967. doi:10.1016/j.mee.2009.11.134

38. Vrij, A. Discuss. Faraday Soc. 1996, 42, 23-33. doi:10.1039/df9664200023

39. Mullins, W. W. J. Appl. Phys. 1957, 28, 333-339. doi:10.1063/1.1722742

40. Mullins, W. W. Acta Metall. 1958, 6, 414-427. doi:10.1016/0001-6160(58)90020-8

41. Müller, C. M.; Spolenak, R. Acta Mater. 2010, 58, 6035-6045. doi:10.1016/j.actamat.2010.07.021

42. Thompson, C. V. Annu. Rev. Mater. Sci. 2000, 30, 159-190. doi:10.1146/annurev.matsci.30.1.159

43. Palmer, J. E.; Thompson, C. V.; Smith, H. I. J. Appl. Phys. 1987, 62, 2492-2497. doi:10.1063/1.339460

\section{License and Terms}

This is an Open Access article under the terms of the Creative Commons Attribution License

(http://creativecommons.org/licenses/by/2.0), which permits unrestricted use, distribution, and reproduction in any medium, provided the original work is properly cited.

The license is subject to the Beilstein Journal of

Nanotechnology terms and conditions:

(http://www.beilstein-journals.org/bjnano)

The definitive version of this article is the electronic one which can be found at: doi:10.3762/bjnano.2.37 